\title{
New observations of cool carbon stars in the halo ${ }^{\star}$
}

\begin{abstract}
N. Mauron
GRAAL, CNRS and Université Montpellier II, Place Bataillon, 34095 Montpellier, France

e-mail: mauron@graal.univ-montp2.fr

Received 10 January 2008 / Accepted 9 February 2008

ABSTRACT

Aims. We report new results of our search for rare, cool carbon stars located at large distances from the Galactic plane. Methods. Candidate stars were selected in the 2MASS point source catalogue with $J H K_{\mathrm{s}}$ colours typical of N-type carbon stars, with $K_{\mathrm{s}} \gtrsim 6.0$ and with Galactic latitude $|b|>20^{\circ}$. Low resolution slit spectroscopy was carried out on 58 candidates.

Results. Eighteen new carbon stars were discovered. Six are remarkable by showing the two peculiarities of a strong infrared excess at $12 \mu \mathrm{m}$ and a large height above the Galactic plane, from 1.7 to $6 \mathrm{kpc}$. The number of $\mathrm{C}$ stars with these properties has been increased to 16 . Mass-loss rates were tentatively estimated by assuming that all these 16 stars are Miras and by using the correlation between $\dot{M}$ and the $K-[12]$ colour index. It is found that several stars have large mass loss, with a median $\dot{M}$ of $4 \times 10^{-6} M_{\odot} \mathrm{yr}^{-1}$ and a dispersion of about a factor of 3 around this value. It would be desirable to detect their CO emission to see whether, like one object already on the list, they display a very low expansion velocity that could be the signature of AGB mass loss at low metallicity. The distances of our new carbon stars were determined by supposing them to be similar to those of the Sagittarius dwarf galaxy. These distances are relatively uncertain, but they do indicate that eight stars might be more than $30 \mathrm{kpc}$ from the Sun, and two at the unprecedented distance of $150 \mathrm{kpc}$.
\end{abstract}

Key words. stars: carbon - stars: mass-loss - Galaxy: halo - Galaxy: stellar content

\section{Introduction}

Carbon (C) stars have been the subject of many studies, in particular because of their rich diversity. Carbon stars are also used for investigating the global properties of galaxies such as their metallicity and star-forming history, the enrichment of their interstellar medium in carbon and carbon-rich dust, or the metalpoor stellar populations in the halos. With considerable simplification, one can say that one type of $\mathrm{C}$ star is composed of red, luminous, variable stars on the asymptotic giant branch (AGB) for which carbon is synthesized in the core and dredged up to the stellar outer layers. These stars are the N-type stars. Another family of $\mathrm{C}$ stars comprises binary stars where one of the components has already passed through the AGB phase and polluted the visible component with carbon-rich material. Among these objects are the $\mathrm{CH}$-type giants and the carbon dwarfs, which are generally older than N-type objects (for a review of carbon stars, see Wallerstein \& Knapp 1998).

This paper is centred on the search for new N-type stars that are located in the Galactic halo and do not belong to the Galactic thin disc like the large majority of known cool C stars. Bothun et al. (1991) considered a sample of 32 faint $C$ stars in the halo and noted that a few of these stars in the Southern hemisphere were very red, indicating an intermediate-age population. Totten $\&$ Irwin (1998) carried on the census and discovery of faint, high Galactic latitude C stars by selecting very red objects on Schmidt plates, which resulted in a list of $77 \mathrm{~N}$-type or $\mathrm{CH}$-type objects. Because the N-type stars were unlikely to have formed in the halo, Totten \& Irwin propose that they come from the disruption of tidally captured dwarf satellite galaxies. Subsequently, Ibata et al. (2001) demonstrate that half of these 77 objects originate

^ Based on observations done at Haute Provence Observatory operated by the Centre National de Recherche Scientifique (France). in the Sagittarius dwarf galaxy, and are part of the tidal debris left by this galaxy in its orbital path around the Milky Way.

The goal of this work is to continue the exploration of the halo for new cool C stars and to make a complete census of them, at least at Galactic latitude $|b| \gtrsim 20^{\circ}$. The driving idea of this exploration is to exploit the 2MASS catalogue of point sources (Cutri et al. 2003). A first reason is that cool C stars are very strong near-infrared objects, typically with $K_{\mathrm{s}}=13$ at $100 \mathrm{kpc}$. Secondly, cool C stars occupy a restricted region in the colourcolour diagram, which helps in finding good $\mathrm{C}$ candidates (see e.g. Fig. 3 of MacConnell 2003). Following our previous investigations (Mauron et al. 2004, 2005, 2007), two aspects appeared particularly interesting. One aspect concerns the cool C stars that one can detect at a very large distance from the Galactic centre $(\gtrsim 100 \mathrm{kpc})$. These stars are the most luminous of a stellar system (e.g. a stream) that still comprises a small population of stars of intermediate age. Therefore, their presence might help for finding where the more numerous but fainter giants of this system would lie, approximatively. Consequently, it seemed important to us to discover N-type stars as far as possible within a large volume of the Galactic halo.

The second aspect of our search for new N-type stars in the halo concerns stellar evolution and, in particular, mass loss of AGB stars at low metallicity. According to Zijlstra (2004), there is indirect evidence that the mass-loss efficiency for $[\mathrm{Fe} / \mathrm{H}]<-1$ is very low. But even for $0<[\mathrm{Fe} / \mathrm{H}]<-1$, the mechanism and the strength of this mass loss is more poorly documented than for AGB stars in the solar vicinity. One of the reasons for this situation is that metal-poor AGB stars in the Magellanic Clouds are too distant for the circumstellar gas (e.g. CO) to be detected with current millimetric instrumentation. However, a small number of very red N-type C stars are located far from the Galactic plane (Groenewegen et al. 1997; Mauron et al. 2007) which makes 
Table 1. List of discovered carbon stars and their characteristics.

\begin{tabular}{|c|c|c|c|c|c|c|c|c|c|c|c|}
\hline No. & 2MASS name & $l$ & $\bar{b}$ & $B$ & $R$ & $B-R$ & $\bar{J}$ & $H$ & $\bar{K}_{\mathrm{s}}$ & $J-K_{\mathrm{s}}$ & Note \\
\hline 84 & 2MASS J003632.34-225451.0 & 83.435 & -84.602 & 19.4 & 17.4 & 2.0 & 15.473 & 14.596 & 14.144 & 1.329 & \\
\hline 85 & 2MASS J021926.96+355058.9 & 142.299 & -23.690 & 21.2 & 14.4 & 6.8 & 11.719 & 9.914 & 8.518 & 3.201 & 1 \\
\hline 86 & 2MASS J022431.98+372933.0 & 142.664 & -21.786 & 21.9 & 16.3 & 5.6 & 11.489 & 9.908 & 8.753 & 2.736 & 1,3 \\
\hline 87 & 2MASS J023904.88+345507.5 & 146.697 & -22.937 & 19.0 & 16.6 & 2.4 & 12.264 & 10.151 & 8.386 & 3.878 & 1 \\
\hline 88 & 2MASS J035955.96+091904.4 & 181.024 & -31.584 & 19.0 & 15.6 & 3.4 & 12.757 & 11.229 & 10.114 & 2.643 & 1 \\
\hline 89 & 2MASS J112705.59+101540.4 & 249.238 & +63.941 & 17.1 & 14.3 & 2.8 & 13.144 & 12.260 & 11.673 & 1.471 & \\
\hline 90 & 2MASS J130118.47+002950.7 & 308.423 & +63.264 & 21.2 & 18.1 & 3.1 & 15.144 & 14.106 & 13.513 & 1.631 & 1 \\
\hline 91 & 2MASS J133557.07+062354.9 & 331.973 & +66.727 & 19.1 & 15.0 & 4.1 & 12.270 & 11.195 & 10.575 & 1.695 & \\
\hline 92 & 2MASS J144644.19+051238.0 & 359.497 & +54.876 & 18.7 & 14.3 & 4.4 & 13.637 & 12.287 & 11.349 & 2.288 & \\
\hline 93 & 2MASS J163631.69-032337.4 & 12.734 & +27.794 & 20.0 & 13.6 & 6.4 & 10.004 & 7.897 & 6.098 & 3.906 & 2 \\
\hline 94 & 2MASS J172046.20-000423.5 & 22.082 & +20.023 & 15.6 & 12.3 & 3.3 & 9.522 & 8.582 & 8.212 & 1.310 & \\
\hline 95 & 2MASS J172554.39+030026.5 & 25.619 & +20.363 & 19.4 & 16.1 & 3.3 & 14.794 & 13.889 & 13.409 & 1.385 & \\
\hline 96 & 2MASS J181329.45+453117.5 & 73.053 & +25.349 & 18.2 & 13.6 & 4.6 & 10.519 & 8.489 & 6.710 & 3.809 & \\
\hline 97 & 2MASS J184950.90+621725.4 & 92.339 & +23.989 & 15.6 & 12.2 & 3.4 & 8.804 & 7.972 & 7.495 & 1.309 & \\
\hline 98 & 2MASS J195652.65-140520.5 & 27.282 & -20.741 & 17.7 & 15.7 & 2.0 & 13.781 & 12.932 & 12.478 & 1.303 & \\
\hline 99 & 2MASS J200303.83-194903.9 & 22.189 & -24.332 & 16.9 & 13.1 & 3.8 & 10.531 & 9.633 & 9.110 & 1.421 & \\
\hline 100 & 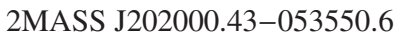 & 38.153 & -22.230 & 19.4 & 16.0 & 3.4 & 12.044 & 10.190 & 8.613 & 3.431 & 1 \\
\hline 101 & 2MASS J204817.91+102638.7 & 56.823 & -20.154 & 20.6 & 15.2 & 5.4 & 10.059 & 8.786 & 7.830 & 2.229 & \\
\hline
\end{tabular}

Notes: (1) the $B$ and $R$ magnitudes are from the USNO-B1.0 catalogue; (2) the $B$ mag is a lower limit (see text); (3) this star was also identified to be a C star by Cruz et al. (2007).

them interesting to consider. If they belong to a tidal stream or if they are members of the thick disc, there is a significant probability that some of them might be metal-poor. At least two of them show signs of oxygen underabundance (Groenewegen et al. 1997). Therefore, these objects are key targets for investigating mass-loss at low metallicity. In this paper, we discuss our discovery of 6 new, very red, mass-losing $\mathrm{C}$ stars with distances from the Galactic plane between 1.7 and $6 \mathrm{kpc}$.

In the following sections, we report new observations of 58 candidate $\mathrm{C}$ stars in the halo. This represents the last observational part of our programme. The general method of candidate selection was explained in Mauron et al. (2004) so is not repeated here, but some new considerations are given in Sect. 3.1. In Sect. 2, the observations and data reductions are described. In Sect. 3, the results of our observations are analysed, especially the spectra, the IRAS counterparts, and an estimation of massloss rates. Section 3.4 concerns the distances and the location of our stars in the halo. Final conclusions and suggestions are given in Sect. 4.

\section{Observations and reductions}

The observations were made during the nights May 16 to 21 and September 10 to 14, 2007 at the Haute-Provence Observatory. The 1.93-m telescope was equipped with the CARELEC spectrograph. This instrument was used with a $150 \mathrm{~g} \mathrm{~mm}^{-1}$ grating. The detector is an EEV $2048 \times 1024$ CCD chip with $13.5 \times 13.5 \mu \mathrm{m}$ pixels. The resulting dispersion is $3.6 \AA$ per pixel. The slit width is $2.0 \mathrm{arcsec}$. The resolving power is $\lambda / \delta \lambda=460$, and $\delta \lambda=13 \AA$ at $6000 \AA$, with a spectral coverage of 4400 to $8400 \AA$. This low resolution was chosen to allow spectroscopy of some targets as faint as $(R \sim 17-18)$ for which the exposure time was $\sim 1 \mathrm{~h}$. In these spectra, even if the signal-to-noise ratio is low, it is relatively easy to see the molecular bands of $\mathrm{C}_{2}$ and $\mathrm{CN}$ typical of cool carbon stars. When the sky transparency was poor and/or the seeing was not good, spectroscopy was achieved for brighter targets with $R \sim 13-14$. We also took spectra of 23 supplementary, relatively bright, candidate $\mathrm{C}$ stars detected in the Byurakan objective-prism survey (Gigoyan et al. 2001). These objects needed slit spectroscopy for confirmation of their carbon-rich nature. All these supplementary spectra are given in the Appendix.

The reductions of the CCD frames included bias subtraction, flat-fielding, extraction of one-dimensional spectra (object and sky), sky subtraction, cleaning of cosmic rays hits, and wavelength calibration. The instrumental spectral efficiency was corrected with the spectrum of a standard photometric star observed during one night. No correction for atmospheric extinction was attempted. The obtained spectra are proportional to a signal expressed in $\operatorname{erg~s}^{-1} \mathrm{~cm}^{-2} \AA^{-1}$. However, no absolute calibration could be achieved because the sky was usually not photometric, and because strong slit losses occurred when the seeing was poor. Therefore, only relative spectral distributions are drawn in our plots.

\section{Results}

Table 1 lists the main parameters of the 18 objects that are under analysis in this work. The columns of Table 1 give the running number following those of Mauron et al. (2007), the 2MASS name comprising the J2000 coordinates coded as JHHMMSS.ss+DDMMSS.s, the Galactic coordinates $l$ and $b$ in degrees, the $B$ and $R$ magnitudes and the $B-R$ colour index, the $J H K_{\mathrm{S}}$ magnitudes from the 2MASS point-source catalogue and the $J-K_{\mathrm{s}}$ colour index.

The $B$ and $R$ magnitudes are from the USNO-A2.0 catalogue of Monet et al. (1998). When no such data was provided, we considered the data of the USNO-B1.0 catalogue (Monet et al. $2003)$, which is indicated in the Notes of Table 1 . When two $B$ or $R$ magnitudes are given in USNO-B1.0, the average is adopted. For star \#93, there is no available information for the $B$ magnitude in either catalogue. Therefore, we adopted from USNOA2.0 the $B$ magnitude of the faintest object within a radius of 2.5 arcmin, giving $B \approx 20.0$. The resulting $B-R$ (in fact a lower limit) is 6.4. This is consistent with the strong $J-K_{\mathrm{s}}$ colour. An additional indication that $B-R$ must be very large is the fact that its spectrum strongly rises to the red (see below).

These $B$ and $R$ magnitudes are not very accurate, with an uncertainty of $\sim 0.4 \mathrm{mag}$, but they nevertheless give useful information. For example, it can be noted that out of 18 objects, 
14 have $B-R \geq 3.0$, which agrees with the typical colour of N-type stars. We also note that object \#84, the faintest in the $K_{\mathrm{s}}$-band and the most distant in our list (see below), has $B-R=2.0$ and might have been eliminated in the list of 2MASS candidates if $B-R$ had been required to be larger.

Two objects, \#84 and \#90, are very faint in the $K_{\mathrm{s}}$-band, with $K_{\mathrm{s}}=14.14$ and $K_{\mathrm{s}}=13.51$, respectively. In previous studies, we had not attempted to detect objects as faint as that, and we had limited our search for $\mathrm{C}$ stars among candidate objects having $K_{\mathrm{s}}<13.5$. For the observations described here, we extended our selection of candidates to $K_{\mathrm{s}}=14.5$. This needed to eliminate a very large number $(2500)$ of contaminating galaxies that have $J H K_{\mathrm{s}}$ colours similar to $\mathrm{C}$ stars. These galaxies are present in the 2MASS point-source catalogue despite their obviously appearing as diffuse objects in the digitized POSS plates. This search for faint, good quality candidates resulted in finding 24 best cases with $K_{\mathrm{s}}$ between 13.5 and $14.5, \delta \geq-25^{\circ}$ and $R \lesssim 18.0$. The $R$-band constraint was applied to allow spectroscopy to be carried out with our instrumentation. Of these 24 objects, ten were observed: two were carbon stars, six M-type stars, and the remaining two were difficult to classify, but certainly not carbon-rich. This suggests that several very distant C stars remain to be discovered with $R \lessgtr 18.0$ in the northern hemisphere, but probably not a large number. A more sensitive instrumentation than the CARELEC spectrograph is needed to search for $\mathrm{C}$ stars beyond $R=18$.

One source has already been classified as a carbon star: object \#93 is CGCS 3716 in the catalogue of Galactic carbon stars of Alksnis et al. (2001). This object is IRAS 16339-0317. LittleMarenin et al. (1987) detected the SiC $11.2 \mu \mathrm{m}$ feature in its IRAS low resolution spectra. Epchtein et al. (1990) also classified it as a carbon star by considering its location in combined IRAS and $J H K L$ colour-colour diagrams that separate oxygenrich and carbon-rich giants. Our spectrum firmly establishes the star to be carbon-rich by showing a very clear $\mathrm{CN}$ molecular band. Another source (\#97) is known as a variable star of the Mira Ceti type (Dahlmark 1998; Kazarovets et al. 2000). Its name is IZ Dra and its period 312 days. Our observations show that it is carbon-rich.

Finally, it can be noted that two objects (\#95 and \#98) that are quite faint in $K_{\mathrm{s}}(\sim 13)$, were found near the limit of our survey in Galactic latitude, at $b=+20.3^{\circ}$ and at $b=-20.7^{\circ}$. We shall see that these objects have distances of about 100 and 60 kpc, respectively, with \#95 remarkable because of its large distance to the main plane of the Sgr Stream. Traditionally, faint high-latitude carbon stars were searched for at $|b| \gtrsim 30^{\circ}$, but our experiment shows that searching for distant $\mathrm{C}$ stars closer to the Galactic plane is feasible.

\subsection{Spectra}

All obtained spectra (except the three in Fig. 1) are presented in the Appendix. In Fig. 1, we show three representative spectra. Object \#89 is relatively bright $(R=14.3)$ so that the signal to noise ratio is high; this star is moderately red in the near-infrared $\left(J-K_{\mathrm{s}}=1.47\right)$. Object \#84 is the faintest in $K_{\mathrm{s}}$ with $K_{\mathrm{s}}=14.14$ and $J-K_{\mathrm{s}}=1.33$. Its spectrum has the lowest $\mathrm{S} / \mathrm{N}$ in our sample but clearly shows the $\mathrm{C}_{2}$ bands at $\lambda<5600 \AA$. Finally, object $\# 100$ is one of the reddest sources $\left(J-K_{\mathrm{s}}=3.43\right)$, displaying a strongly rising continuum, with the $\mathrm{CN}$-band at $7800 \AA$ deep enough to prove its carbon-rich nature.

Many objects display $\mathrm{H} \alpha$ in emission. For several objects, this emission is faint $(\# 84,85,87,94)$, while for others the
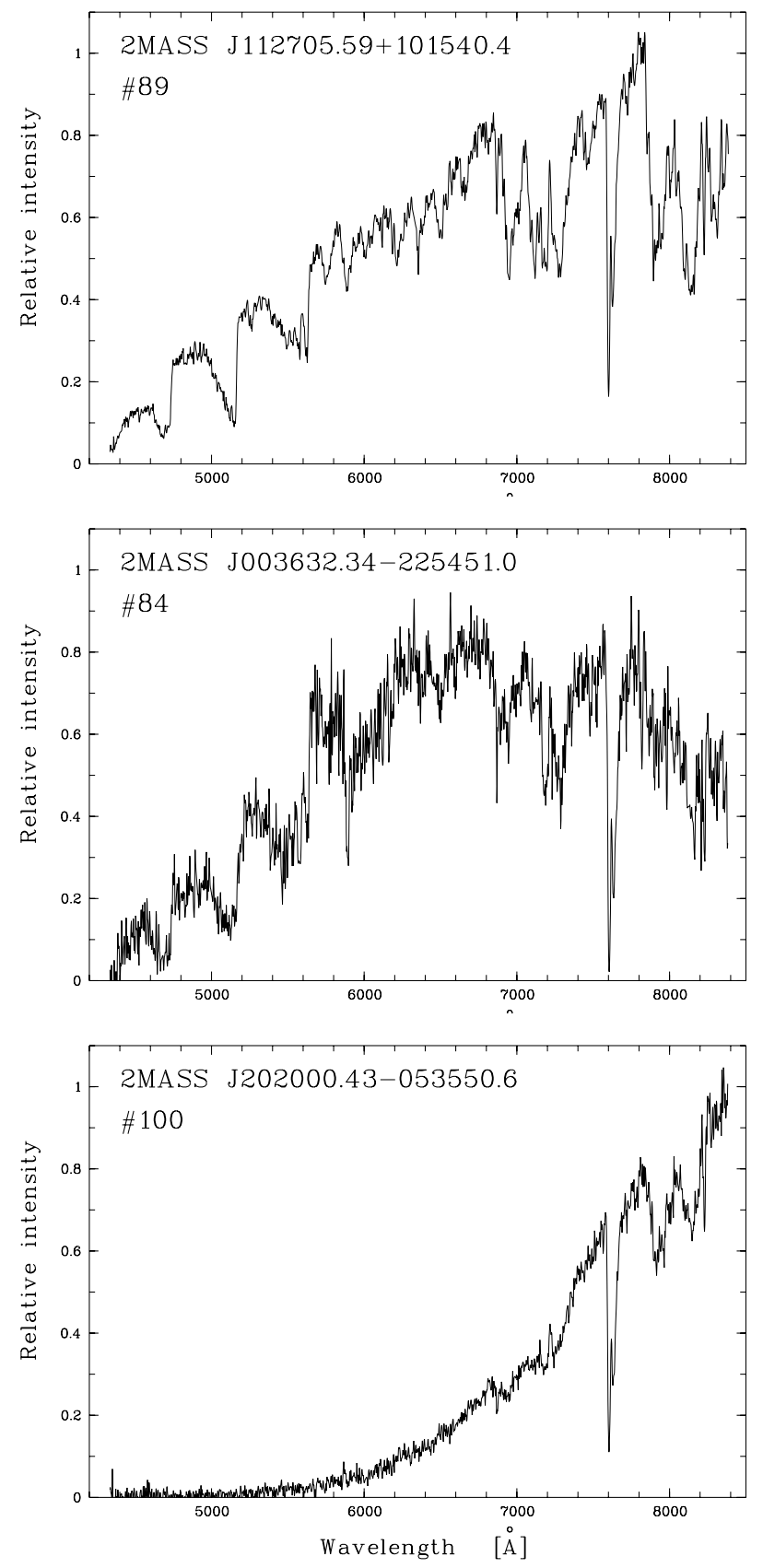

Fig. 1. Three typical spectra of our sample. Top panel: object \#89, a relatively bright source, $R=14.3$, with $J-K_{\mathrm{s}}=1.47$. Middle panel: object \#84, the faintest source in $K_{\mathrm{s}}$ with $K_{\mathrm{s}}=14.14, R=17.4, J-$ $K_{\mathrm{s}}=1.33$. Bottom panel: object \#100, a very red source in $J-K_{\mathrm{s}}$, with $R=16.0, K_{\mathrm{s}}=8.61, J-K_{\mathrm{s}}=3.4$.

emission is strong (\#86, 88, 90, 91, 96, 97, 98). The fraction of stars with this emission line is therefore $\sim 50 \%$, in fair agreement with our previous studies. There is one object, \#97, for which $\mathrm{H} \beta$ and $\mathrm{H} \gamma$ in emission are also visible. Finally, object \#96 shows $\mathrm{H} \alpha, \mathrm{H} \beta$, and [O III] $\lambda 5007 \AA$ in emission and might be a symbiotic star with a hot ionizing companion.

\subsection{Objects with IRAS counterparts}

For all objects in Table 1, we searched for IRAS counterparts and found eight cases, with six sources from the IRAS point-source catalogue (Beichman et al. 1989) and two additional sources 
from the IRAS faint source catalogue (Moshir et al. 1989). The results are in Table 2 . In this table, one finds the names and the galactic coordinates, the IRAS $12 \mu \mathrm{m}$ flux, $K_{\mathrm{s}}$ and $J-K_{\mathrm{s}}$ from 2MASS, the distances to the Sun $d$ and the heights $Z$ above the Galactic plane (see below for details on the derivations of $d$ and $Z$ ). There are five objects with $J-K_{\mathrm{s}}>3$ that have counterparts with $12-\mu$ m fluxes $\left(f_{12}\right)$ between $\sim 0.4$ and $14 \mathrm{Jy}$. The three other objects with smaller $J-K_{\mathrm{s}}$ generally have lower $f_{12}$. No star with $K_{\mathrm{s}} \gtrsim 8.8$ was found to be detected by IRAS.

In Table 2, one can see that the distances from the Galactic plane are between 1.7 to $6 \mathrm{kpc}$. This indicates that these sources do not belong to the Galactic thin disc, since the scale height of disc carbon stars is only 200 pc (Claussen et al. 1987; Groenewegen et al. 1992; Bergeat et al. 2002). It would be important to measure the kinematics of these stars. This would allow us to see whether they might belong to the thick disc (although this disc is rather old for dusty AGB C stars to be present) or are members of a stream in the Galactic halo, especially the Sgr Stream.

These dusty $\mathrm{C}$ stars deserve attention because their $Z$ are larger than most of the AGB C stars known. Compared to $C$ stars of the Galactic disc, their circumstellar envelopes will have a different environment, probably with a lower interstellar density, and weaker ultraviolet ambient radiation from hot stars of the Galactic disc. Therefore, it is quite possible that the extension of the $\mathrm{CO}$ emitting envelope will be unusually large. For a typical mass-loss rate of $4 \times 10^{-6} M_{\odot} \mathrm{yr}^{-1}$ (the median mass loss rate in Table 3, see below), the $\mathrm{CO}$ photodissociation radius for AGB C stars in the disc is about 0.05 pc (from equation 7.9 of Olofsson 2004). If it is twice larger for our high $Z$ stars ( $\sim .1 \mathrm{pc})$, this corresponds, at a median distance of $\sim 10 \mathrm{kpc}$, to an angular diameter of $4^{\prime \prime}$, which should be easily detected and resolved by future millimeter interferometers like ALMA.

\subsection{Mass-loss rates}

For stars with available IRAS $12-\mu$ m fluxes, it is interesting to see what could be their mass-loss rates, even if it is only estimated approximatively. In a study of Galactic, Mira-type carbon stars, Whitelock et al. (2006) derived a tight correlation between the $K$-[12] colour and the total mass-loss rate $\dot{M}$. In this correlation, $K$ is the mean magnitude (averaged over time), and [12] is the IRAS magnitude defined as $-2.5 \log _{10}\left(f_{12} / 28.3\right)$ where $f_{12}$ is in Jy. For a given $K$-[12], $\dot{M}$ is given by: $\log \dot{M}=$ $-7.668+0.7305(K-[12])-5.398 \times 10^{-2}(K-[12])^{2}+1.343 \times$ $10^{-3}(K-[12])^{3}$. For establishing this correlation, Whitelock et al. calculated $\dot{M}$ by using the relation of Jura (1987) where $\dot{M}$ depends on the IRAS $60-\mu$ m flux, on the distance of the object, on the wind velocity, and on several other parameters of the circumstellar shells (see Eq. (11) in Whitelock et al. 2006). In particular, the Jura relation assumes a gas-to-dust mass ratio of 220 (Jura 1986).

Whitelock et al. (2006) noted that the correlation between $\dot{M}$ and $K-[12]$ established for Galactic C stars is also verified for the carbon Miras of the Large Magellanic Cloud. In this case, the LMC C Miras were taken from the work by Van Loon et al. (1999) who adopt a gas-to-dust ratio of 500. Consequently, the $K-$ [12] versus $\dot{M}$ relation holds, provided one supposes that there is less dust produced in the winds of $\mathrm{C}$ stars at lower metallicity, which is also favoured by recent Spitzer observations of C stars in external galaxies (e.g. Matsuura et al. 2007).

Although we do not know the metallicity of our sources, we suppose that the Whitelock et al. relation can be applied to them. We must also assume that our stars are Mira variables and that the 2MASS $K_{\mathrm{s}}$ magnitude is not very different from $K$ averaged over time (if the difference is $\sim 0.5 \mathrm{mag}$, it will change $\dot{M}$ by only 40 percent). With these assumptions, we obtain the results of Table 3, in which $\dot{M}$ is estimated from the $K-$ [12] colour and is uncertain by about a factor of 2 . The first six objects are from this work. The following ten objects, designated by their IRAS names, are objects with $J-K_{\mathrm{s}}>3$ extracted from Table 2 of Mauron et al. (2007). In Table 3, we have not included Objects \#97 and \#101 which are relatively blue with small $J-K_{\mathrm{s}}$ indices and $K-$ [12] in the range of 2.0-2.3, making the assumption of Mira-type variability more questionable. The distances and height above the Galactic plane are also mentioned in Table 3, but it must be emphasized that the $\dot{M}$ values that we obtained above do not depend on $d$ or $Z$ since they are directly derived from $K-$ [12].

Table 3 shows that, presently, we have identified $16 \mathrm{C}$ stars out of the Galactic plane, with 6 coming from this work. Several objects have $\dot{M}$ of the order of $4 \times 10^{-6} M_{\odot} \mathrm{yr}^{-1}$ or even larger. It can be noted that both the distances and the mass-loss rates have been derived homogeneously for all of them, so that useful comparisons can be made between objects. For example, one may consider IRAS $12560+1656$ as a reference object since it was detected in $\mathrm{CO}$ with such a low and unusual expansion velocity of $3 \mathrm{~km} \mathrm{~s}^{-1}$ probably due to its low metallicity. Then, a look at Table 3 shows that six objects have larger $\dot{M}$, together with smaller distances $d$. These six cases should be easier to detect in CO than IRAS $12560+1656$.

\subsection{Distances and positions in the halo}

The distances of our new $\mathrm{C}$ stars are a priori difficult to estimate because they are field stars. We follow the method described by Mauron et al. (2004) and adopted in our previous works on halo $\mathrm{C}$ stars, but we provide here more detailed information. Only near infrared data from 2MASS ( $J$ and $K_{\mathrm{s}}$ ) are used because they are less affected than optical data by interstellar or circumstellar extinction, or by variability. The $B$ or $R$ magnitudes are not used.

The first step in the method consists in obtaining $K_{\mathrm{s}}$-band absolute magnitudes for $\mathrm{C}$ stars of the LMC. The catalogue of LMC optically-selected C stars of Kontizas et al. (2001) provides a first sample of $\mathrm{C}$ stars to consider. In particular, this sample contains $\sim 5000 \mathrm{C}$ stars with colours between $J-K_{\mathrm{s}}=1.0$ and $J-K_{\mathrm{s}}=2.0$. A second sample of C stars is obtained by considering the regions labelled $\mathrm{J}$ and $\mathrm{K}$ in the 2MASS colour-magnitude diagrams, as defined by Nikolaev \& Weinberg (2000, hereafter NW00; see their Fig. 3). Region J contains $~ 7000$ objects with $1.4<J-K_{\mathrm{s}}<2.0$, and region K contains 1600 objects (often called obscured AGB stars) with $2.0<J-K_{\mathrm{s}}<4.0$. According to NW00 (their Fig. 8), the objects located in these two regions are in a large majority, but not exclusively, carbon stars. Several works in the past have found OH/IR stars in region K. For example, Wood et al. (1992) searched for OH emission on 54 very red objects and found it for 6 objects. Five of them have near infrared photometry, and two are in region K. Whitelock et al. (2003) also considered obscured C-rich and O-rich AGB stars in the LMC: of the 19 O-rich objects with available $J H K$ photometry, 7 are in region K. But more recent work based on $J H K, 8 \mu \mathrm{m}$ photometry, and Spitzer infrared spectroscopy suggest that the overwhelming majority of AGB stars are carbon stars (Buchanan et al. 2006). Also, among the 250 brightest and compact $8 \mu \mathrm{m}$ sources in the LMC, Kastner et al. (2007) find that there are 7 times more C-rich AGB stars than O-rich AGB stars. By considering the 2MASS photometry of these 250 sources (from their Table 1), we find that region $\mathrm{K}$ contains $41 \mathrm{CAGB}$ stars, 
Table 2. Objects of Table 1 with IRAS counterparts.

\begin{tabular}{|c|c|c|c|c|c|c|c|c|c|c|}
\hline \# & IRAS name & 2MASS name & $\begin{array}{c}l \\
(\mathrm{deg})\end{array}$ & $\begin{array}{c}b \\
(\mathrm{deg})\end{array}$ & $\begin{array}{r}f_{12} \\
(\mathrm{Jy})\end{array}$ & $\begin{array}{c}K \\
\text { (mag) }\end{array}$ & $\begin{array}{l}J-K \\
(\mathrm{mag})\end{array}$ & $\begin{array}{c}d \\
(\mathrm{kpc})\end{array}$ & $\begin{array}{c}Z \\
(\mathrm{kpc})\end{array}$ & Note \\
\hline$\# 85$ & $02164+3537$ & J021926.96+355058.9 & 142.299 & -23.690 & 0.41 & 8.52 & 3.20 & 13.3 & -5.3 & \\
\hline \#86 & F02214+3716 & J022431.98+372933.0 & 142.664 & -21.786 & 0.16 & 8.75 & 2.74 & 16.2 & -6.0 & 1 \\
\hline \#87 & $02360+3442$ & J023904.88+345507.5 & 146.697 & -22.937 & 1.16 & 8.39 & 3.89 & 11.0 & -4.3 & \\
\hline \#93 & $16339-0317$ & J163631.69-032337.4 & 12.734 & +27.794 & 14.57 & 6.10 & 3.91 & 3.7 & +1.7 & \\
\hline \#96 & $18120+4530$ & $\mathrm{~J} 181329.45+453117.5$ & 73.053 & +25.349 & 7.86 & 6.71 & 3.81 & 5.1 & +2.2 & \\
\hline \#97 & $18493+6213$ & $\mathrm{~J} 184950.90+621725.4$ & 92.339 & +23.989 & 0.23 & 7.49 & 1.31 & 7.4 & +3.0 & \\
\hline$\# 100$ & 20173-0545 & J202000.43-053550.6 & 38.153 & -22.230 & 0.45 & 8.61 & 3.43 & 13.3 & -5.0 & \\
\hline \#101 & F20458+1015 & $\mathrm{J} 204817.91+102638.7$ & 56.823 & -20.154 & 0.17 & 7.83 & 2.23 & 11.7 & -4.0 & 1 \\
\hline
\end{tabular}

Notes: (1) the IRAS name and flux come from the IRAS Faint Source Catalogue.

Table 3. Halo carbon stars with predicted high mass-loss rates $\dot{M}$ in units of $10^{-6} M_{\odot} \mathrm{yr}^{-1}$.

\begin{tabular}{crrrr}
\hline \hline Name & $\begin{array}{r}K-[12] \\
(\mathrm{mag})\end{array}$ & $\begin{array}{c}d \\
(\mathrm{kpc})\end{array}$ & $\begin{array}{c}Z \\
(\mathrm{kpc})\end{array}$ & $\dot{M}$ \\
\hline$\# 85$ & 3.92 & 13 & -5.3 & 3.0 \\
$\# 86$ & 3.13 & 16 & -6.0 & 1.5 \\
$\# 87$ & 4.92 & 11 & -4.3 & 6.0 \\
$\# 93$ & 5.38 & 3.7 & +1.7 & 8.0 \\
$\# 96$ & 5.32 & 5.0 & +2.2 & 8.0 \\
$\# 100$ & 4.11 & 13 & -5.0 & 3.5 \\
$01582+0931$ & 4.43 & 7 & -5.5 & 4.0 \\
$03242+1429$ & 4.01 & 13 & -7.5 & 3.0 \\
$03582+1819$ & 5.68 & 17 & -7.5 & 10.0 \\
$04188+0122$ & 4.11 & 6 & -3.5 & 3.5 \\
$08427+0338$ & 4.65 & 6 & +2.5 & 5.0 \\
$08546+1732$ & 6.47 & 36 & +21.0 & 14.0 \\
$12560+1656$ & 3.91 & 11 & +11.0 & 3.0 \\
$20005+7737$ & 4.31 & 14 & +5.5 & 4.0 \\
$20176-1458$ & 4.21 & 14 & -6.0 & 3.5 \\
$21064+7749$ & 4.41 & 4 & +1.3 & 4.0 \\
\hline
\end{tabular}

5 O-rich AGB stars, 3 HII regions, and no red supergiants. Although these numbers are small compared to the total population of region $\mathrm{K}$, they constitute an indication that region $\mathrm{K}$ is very probably dominated by carbon stars. Consequently, we assume that this region $\mathrm{K}$ is suitable for determining the $K_{\mathrm{s}}$-band absolute magnitude of very red LMC C stars.

We adopt a mean LMC interstellar extinction $E(B-V)=$ 0.13 (Van den Bergh 2000), and corresponding extinction for the 2MASS photometry are $A_{K \mathrm{~s}}=0.367 E(B-V)$ and $E\left(J-K_{\mathrm{s}}\right)=$ $0.535 E(B-V)$. A distance modulus $m-M=18.50$ is assumed (Van den Bergh 2000; see also Fig. 8 of Clementini et al. 2003).

The resulting absolute magnitude for LMC C stars $M_{K}$ is calculated for bins in the $\left(J-K_{\mathrm{s}}\right)_{0}$ colour and is shown in Fig. 2. The number of stars in the bins is in the range 50 to 1500 . In the colour interval where the two samples mentioned above overlap, the agreement is very good and their average has been adopted. The error bars represent the dispersion of $M_{K}$ in each bin ( $\pm \sigma$ is drawn). These dispersions are about $0.40 \pm 0.05$ mag.

The second point that we consider is how $\mathrm{C}$ stars of the Sgr dwarf galaxy compare in $M_{K}$ with those of the LMC. No complete catalogue of $\mathrm{C}$ stars of Sgr is available, but Whitelock et al. (1999) have published a list of $26 \mathrm{C}$ stars which are radial velocity members of Sgr. The 2MASS photometry of these stars is available. The interstellar reddening was found for each star from the maps of Schlegel et al. (1998), with $E(B-V)$ found to be in the range 0.09 to $0.18 \mathrm{mag}$. A distance modulus of Sgr $m-M=17.0$ was adopted, which is between the 16.9 taken by Majewski et al. (2003) and 17.18 adopted by

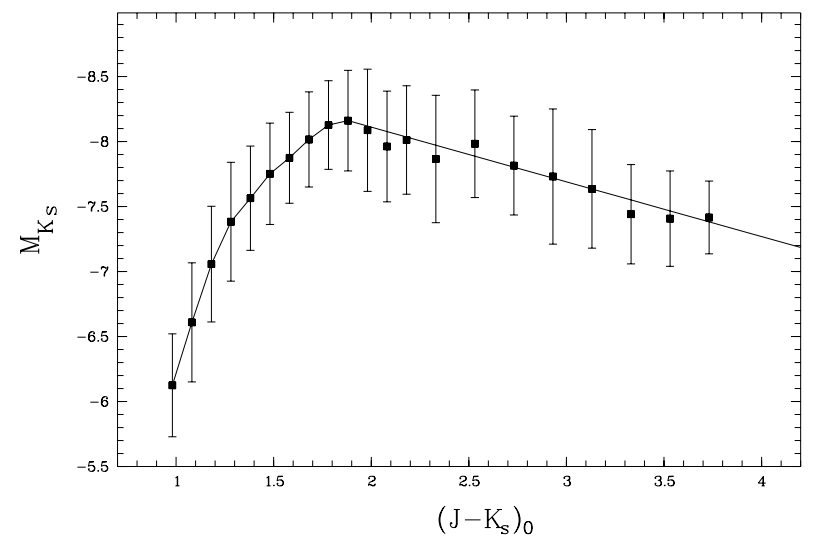

Fig. 2. Absolute magnitudes of the LMC carbon stars in the $K_{\mathrm{s}}$ band as a function of $(J-K)_{0}$ (solid line). The filled squares indicate averaged $M_{K}$ from a given bin in colour, and the error bars indicate the dispersion in each bin ( $\pm \sigma$ is drawn).

Whitelock et al. (1999). This allows us to plot in Fig. 3 the $M_{K \text { s }}$ of these stars versus their $\left(J-K_{\mathrm{s}}\right)_{0}$, and to compare them to the LMC C stars. This figure shows that the Sgr stars are on average 0.5 magnitude less luminous in $K_{\mathrm{s}}$ than the LMC stars. Beyond $J-K_{\mathrm{S}} \sim 2.5$, there are no Sgr points, and we shall assume that the shift of $0.5 \mathrm{mag}$ between LMC and Sgr is still valid in this region. [One can note that this luminosity shift of $0.5 \mathrm{mag}$. between LMC and Sgr C stars is significantly reduced (to $\approx 0.16$ ) if one adopts (1) a closer distance for the LMC, e.g. $m-M=18.39$ from van Leeuwen et al. (2007), or $m-M=18.34$ from An et al. (2007), and (2) a greater distance for Sgr, e.g. $m-M=17.18$ (mentioned above). In this case, the distances of our objects derived below would have to be increased by $\sim 10 \%$ ].

A remark by the referee was that for stars as red as $J-K_{\mathrm{s}} \sim 3$, circumstellar extinction will be significant and should be taken into account when determining distances. To our knowledge, there is no way to measure the circumstellar extinction of a dusty C star, especially when only photometry is available. But its effect is included in the absolute magnitude when we have determined $M_{K}$ for LMC stars: in Fig. 2, the $K_{\mathrm{s}}$-band luminosity decreases beyond $J-K_{\mathrm{s}} \sim 2$ and this is due to circumstellar dust extinction (NW00).

Thus, we assume that our stars are identical to those of the Sgr dwarf galaxy and have similar absolute magnitude in the $K_{\mathrm{s}}$-band for a given $J-K_{\mathrm{s}}$ colour. The distances of our objects are determined by using the LMC $M_{K s}$ values plus 0.5 mag. Interstellar absorption was taken into account although this effect is small, since the largest $E(B-V)$ is $0.38 \mathrm{mag}$. The results are in Table 4. This table lists the running number, the galactic coordinates $l$ and $b$ in degrees, the interstellar colour 
Table 4. Properties of the halo C stars.

\begin{tabular}{rrrrcrrrrrrr}
\hline \hline No. & $l$ & $b$ & $E_{B-V}$ & $\left(J-K_{\mathrm{s}}\right)_{0}$ & $K_{\mathrm{s}}$ & $M_{K \mathrm{~s}}$ & $d$ & $X$ & $Y$ & $Z$ & $D$ \\
\hline 84 & 83.435 & -84.602 & 0.018 & 1.319 & 14.144 & -6.95 & 165 & -6.7 & 15.4 & -164.5 & -22.7 \\
85 & 142.299 & -23.690 & 0.070 & 3.164 & 8.518 & -7.12 & 13 & -18.1 & 7.4 & -5.3 & 7.4 \\
86 & 142.664 & -21.786 & 0.038 & 2.716 & 8.753 & -7.31 & 16 & -20.5 & 9.1 & -6.0 & 9.0 \\
87 & 146.697 & -22.937 & 0.055 & 3.849 & 8.386 & -6.83 & 11 & -16.9 & 5.5 & -4.3 & 5.7 \\
88 & 181.024 & -31.584 & 0.276 & 2.495 & 10.114 & -7.40 & 30 & -34.4 & -0.5 & -15.9 & -1.7 \\
89 & 249.238 & +63.941 & 0.037 & 1.451 & 11.673 & -7.20 & 59 & -17.7 & -24.2 & 53.0 & -9.8 \\
90 & 308.423 & +63.264 & 0.026 & 1.617 & 13.513 & -7.43 & 153 & 34.4 & -54.1 & 137.0 & -22.5 \\
91 & 331.973 & +66.727 & 0.031 & 1.678 & 10.575 & -7.51 & 41 & 5.9 & -7.7 & 37.9 & 1.3 \\
92 & 359.497 & +54.876 & 0.039 & 2.267 & 11.349 & -7.50 & 58 & 25.1 & -0.3 & 47.8 & 9.5 \\
93 & 12.734 & +27.794 & 0.388 & 3.698 & 6.098 & -6.90 & 3.7 & -5.3 & 0.7 & 1.7 & 1.7 \\
94 & 22.082 & +20.023 & 0.315 & 1.141 & 8.212 & -6.38 & 7.9 & -1.7 & 2.8 & 2.7 & 3.7 \\
95 & 25.619 & +20.363 & 0.126 & 1.318 & 13.409 & -6.95 & 115 & 89.1 & 46.8 & 40.2 & 49.3 \\
96 & 73.053 & +25.349 & 0.033 & 3.791 & 6.710 & -6.86 & 5.1 & -7.1 & 4.4 & 2.2 & 5.5 \\
97 & 92.339 & +23.989 & 0.055 & 1.280 & 7.495 & -6.88 & 7.4 & -8.8 & 6.8 & 3.0 & 8.1 \\
98 & 27.282 & -20.741 & 0.264 & 1.162 & 12.478 & -6.47 & 59 & 40.5 & 25.3 & -20.9 & 17.3 \\
99 & 22.189 & -24.332 & 0.164 & 1.333 & 9.110 & -6.98 & 16 & 5.0 & 5.5 & -6.6 & 3.7 \\
100 & 38.153 & -22.230 & 0.074 & 3.391 & 8.613 & -7.02 & 13 & 1.1 & 7.6 & -5.0 & 6.3 \\
101 & 56.823 & -20.154 & 0.078 & 2.187 & 7.830 & -7.53 & 12 & -2.5 & 9.2 & -4.0 & 8.3 \\
\hline
\end{tabular}

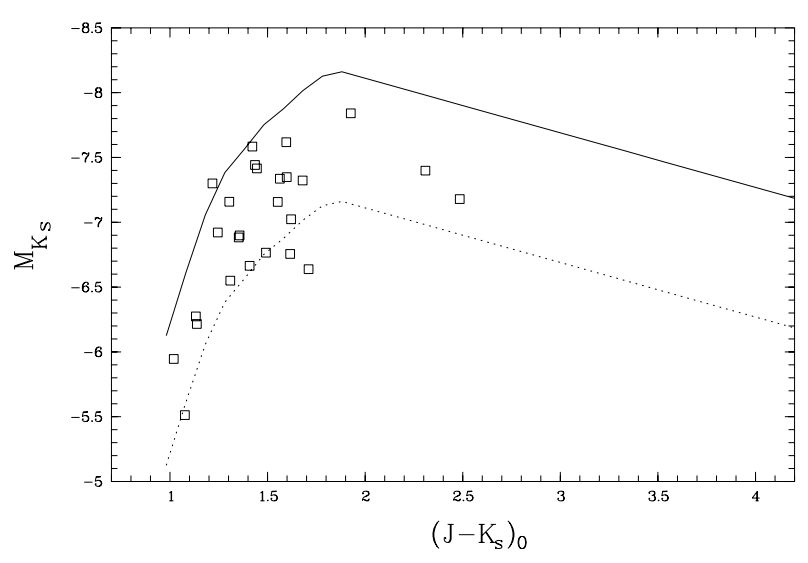

Fig. 3. Comparison of LMC and Sgr absolute magnitudes as a function of colour. The LMC is represented by the solid line as in Fig. 2. The open squares are 26 stars of Sgr. The dotted line is the LMC line shifted down by $1.0 \mathrm{mag}$. On average, the Sgr stars appear to be less luminous than LMC ones by $\sim 0.5 \mathrm{mag}$ (see text).

excess measured in the direction of the object, $E_{\mathrm{B}-\mathrm{V}}$ from Schlegel et al. maps, the dereddened $J-K_{\mathrm{s}}$ colour, the adopted $K_{\mathrm{s}}$-band absolute magnitude, the distance $d$ to the Sun in kpc, the galactocentric $X Y Z$ coordinates in kpc, and the distance $D$ to the Sgr Stream average plane, in kpc. Given the above considerations, the relative uncertainty on these distances is at least of the order of $\pm 20 \%( \pm 1 \sigma)$. In particular, stars having blue colours with $\left(J-K_{\mathrm{s}}\right)_{0} \sim 1.0-1.2$ like \#94 and \#98 have very uncertain distances because there are few points at this colour in Fig. 3, and the slope of $M_{K}$ with colour is very steep.

There are four objects with their distances to the Sun below $10 \mathrm{kpc}, 6$ with $10<d<20 \mathrm{kpc}$, 5 with $20<d<100 \mathrm{kpc}$, and 3 with $d>100 \mathrm{kpc}$. The two objects with the greatest distances are \#84 $(d \approx 165 \mathrm{kpc})$ and $\# 90(d \approx 150 \mathrm{kpc})$. We checked that these distant stars are not carbon dwarfs, especially for the most distant ones (\#84, \#90, \#94). Although the USNOB1.0 catalogue gives a non-zero proper motion for object \#84

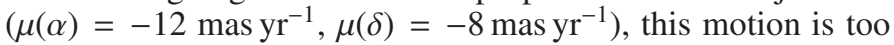
small to be considered with certainty, as was shown for several similar cases in Mauron et al. (2005). The USNO-B1.0 gives zero proper motion for the other two distant objects \#90 and \#95.
An additional suggestion that these objects are AGB stars comes from their $J H K_{\mathrm{s}}$ colours, which put them outside the locus of carbon dwarfs in the colour-colour diagrams shown by Totten et al. (2000) or by Downes et al. (2004).

A check on the distances adopted in this work is possible for a few objects. Object \#97 (IZ Dra) is a Mira variable with a known period (312 days). Then, one can apply the period luminosity $P-K$ of Feast et al. (1989) if one assumes that the 2MASS $K_{\mathrm{S}}$ magnitude is not too different from the time-averaged magnitude. The Feast et al. relation is $K=-3.3 \log P+18.98$ for Miras of the LMC, for which we adopt a distance modulus of 18.50. Therefore, the absolute magnitude $M_{K}$ is given by $M_{K}=-3.3 \log P+0.48$. Then, one obtains $d=11 \mathrm{kpc}$ for IZ Dra, which is more than our estimate $(7.4 \mathrm{kpc})$, but the discrepancy is nearly acceptable.

Our estimates of distances can also be evaluated by considering two objects in Table 3, IRAS 08546+1732 and IRAS $12560+1656$. Their periods are 390 days (see Groenewegen et al. 1997). For the first object, $K_{\mathrm{s}}=10.71$ and the distance derived from the $P-K$ relation is $57 \mathrm{kpc}$, whereas our method gives $36 \mathrm{kpc}$. For the second, $K_{\mathrm{s}}=7.82$ and $15 \mathrm{kpc}$ is obtained, whereas our method gives $11 \mathrm{kpc}$. Therefore, the distances adopted in this work (in Tables 2-4) appear a little too small but still reasonably correct given the uncertainties mentioned above. At least for the reddest objects, a $K$-band monitoring would help to establish their variability and, if they are Miras, to determine these distances more precisely.

We also calculated for Table 4 the galactocentric $X Y Z$ coordinates of each object, as well as their distance $D$ to the main plane of the Sgr Stream as given by Newberg et al. (2003). Plots of the $Y Z$ and $X Z$ are given in Fig. 4. Note that their sizes are $400 \times 400 \mathrm{kpc}$ in order to emphasize the position of very distant objects. In the $Y Z$ panel, the Sgr stream is seen nearly edge-on, slightly inclined clockwise from the $Z$ axis.

The $Y Z$ plot shows that objects \#84 and \#90 are not very far from the Stream plane, only by $22 \mathrm{kpc}$ (see Table 4), which represents an angle of about 8 degrees as seen from the Galactic centre. Object \#84 suggests that the Sgr stream extends to $\sim 150 \mathrm{kpc}$ toward the South pole if our distances are correct, and the qualitative agreement of these observations with the model of Law et al. (2005) concerning the existence of a southern warp is reinforced (see Fig. 4 of Mauron et al. 2005). Object \#90 is, 

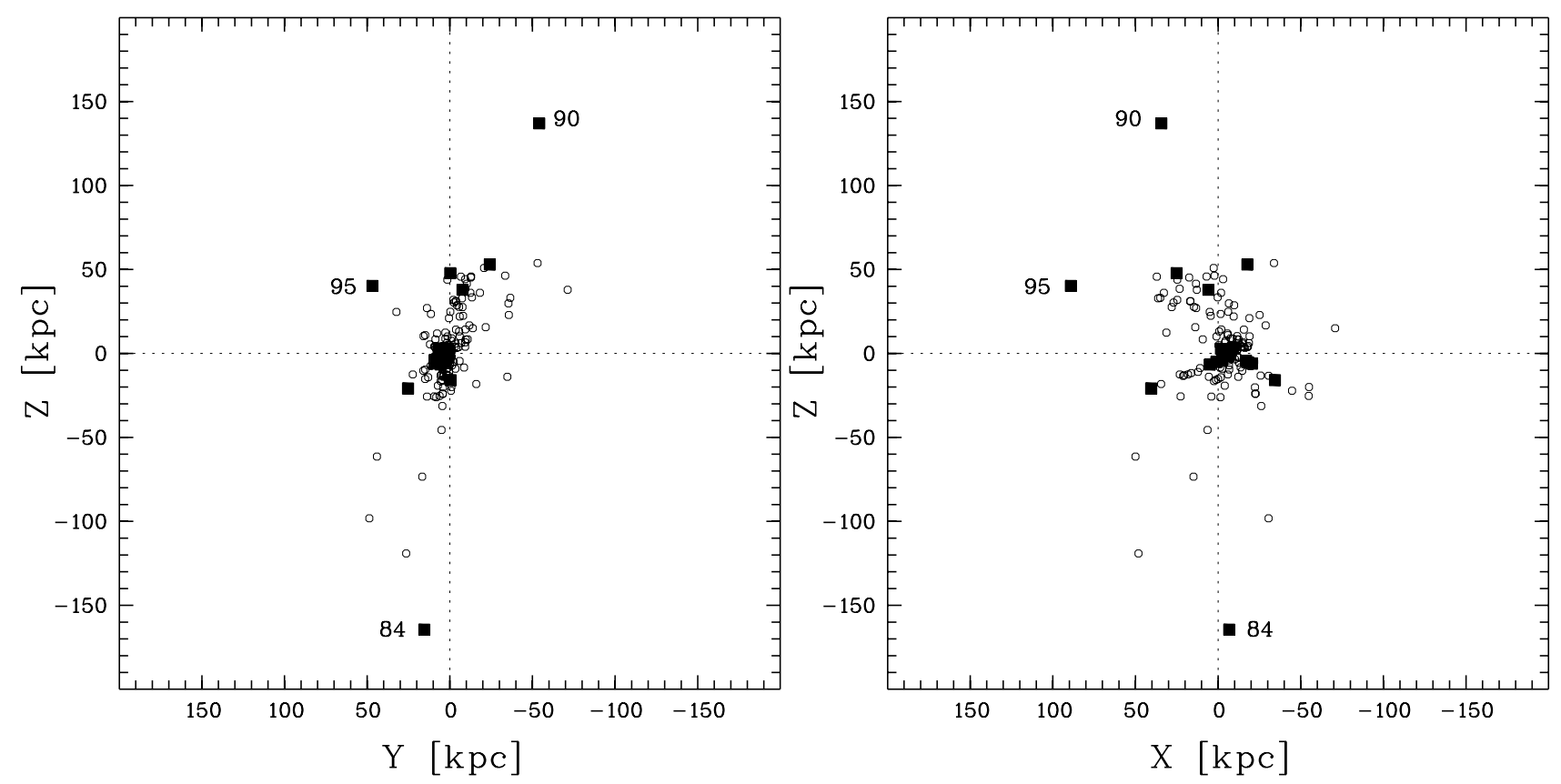

Fig. 4. Plot in galactocentric $X Y Z$ coordinates of the observed halo $\mathrm{C}$ stars, indicated by filled squares. The XYZ system is such that the Sun is at $X=-8.5 \mathrm{kpc}, Y=0, Z=0$; the $Y$-axis is positive towards $l=+90^{\circ}$ and the $Z$ axis is positive to $b=+90^{\circ}$. In the left panel, the Sgr Stream is seen close to edge-on, slightly inclined clockwise with respect to the $Z$ axis. It is seen face-on in the right panel.

in contrast, far toward the North Galactic pole where no warp is predicted. Finally, object \#95 is located by 50 kpc from the Sgr plane and is isolated in this region of the halo, but this may be due in part to its galactic coordinates, $l=25.6^{\circ}, b=+20.3^{\circ}$. At this low latitude, it becomes more difficult to find faint $\left(K_{\mathrm{s}} \sim 13\right)$ carbon stars, because numerous interlopers, such as young stars, come into the candidate list and this decreases the probability of discovering a new faint $\mathrm{C}$ star.

\section{Conclusions and suggestions}

In order to find new cool carbon stars in the halo, we have carried out spectroscopy of 58 candidates selected in the 2MASS catalogue through their near-infrared colours and $|b|>20^{\circ}$. Eighteen new cool carbon stars were discovered. The spectra of these rare stars generally show strong $\mathrm{C}_{2}$ and $\mathrm{CN}$ bands typical of AGB carbon stars, with $\mathrm{H} \alpha$ often in emission, indicating pulsating atmospheres. Of these 18 new $\mathrm{C}$ stars, 10 have IRAS counterparts with detected fluxes at $12 \mu \mathrm{m}$. For 6 of them, the $12 \mu \mathrm{m}$ infrared excess is large, and their heights above the Galactic plane are between 1.7 to $6 \mathrm{kpc}$. After adding these 6 dusty $\mathrm{C}$ stars to previously known cases, a list of 16 similar cases is established (Table 3). This list contains one object (IRAS 12560+1656) for which CO emission was detected (Groenewegen et al. 1997). Its CO line shows a unique expansion velocity of only $3 \mathrm{~km} \mathrm{~s}^{-1}$.

Mass-loss rates $\dot{M}$ have been homogeneously estimated by assuming that the relation between $K-[12]$ and $\dot{M}$ established by Whitelock et al. for Galactic and LMC Mira-type $\mathrm{C}$ stars can be applied to the 16 objects. It is found that the $\dot{M}$ estimates are of the order of $4 \times 10^{-6} M_{\odot} \mathrm{yr}^{-1}$, with a range of a factor of 3 . These strong mass-loss rates suggest that further investigation of these halo AGB stars should be carried out. Several of them might be metal-poor and similar to IRAS 12560+1656, helping us to understand mass loss of AGB C stars with low metallicity better.
Although distances are uncertain by at least $\pm 20 \%$ in relative error $( \pm 1 \sigma)$, taking them at face value shows that three objects might be as far as $100 \mathrm{kpc}$ from the Sun, with two reaching unprecedented distances of $\sim 150 \mathrm{kpc}$. One object (\#84) is located at $165 \mathrm{kpc}$ toward the South Galactic pole and is relatively close, given uncertainties, to a region of the halo where $4 \mathrm{C}$ stars have been discovered previously, and where a loop of the Sgr Stream is predicted. In the future, it would be interesting to measure the radial velocity of these 5 stars and to see if this southern warp can be detected with fainter but much more numerous $\mathrm{M}$ giants in similar regions in the sky and at similar velocities.

Acknowledgements. The author thanks the anonymous referee for useful remarks, and Pauline Mc Nish and Joli Adams for correcting the English of the manuscript. The author also thanks the staff of Observatoire de HauteProvence, which is supported by the French Centre National de Recherche Scientifique. The use of the Two Micron All Sky Survey is acknowledged. 2MASS is a joint project of the University of Massachusetts and the Infrared Processing and Analysis Center/California Institute of Technology, funded by the National Aeronautics and Space Administration (NASA) and the National Science Foundation (NSF). Finally, this work benefitted from using the CDS database in Strasbourg (France).

\section{Appendix A: Spectra of halo C stars}

This appendix presents in a first part the spectra of 15 stars discussed in this paper. The three spectra for objects \#84, \#89, and \#100 are in Fig. 1. The 23 spectra that follow are low-resolution spectra that were needed for confirming candidate carbon stars found in the First Byurakan Survey (FBS) and listed in Gigoyan et al. (2001) or in Mauron et al. (2007). The names of the sources and the J2000 coordinates are given in the plots. There are 10 FBS objects, originally carbon star candidates, that were found to be M-type dwarfs. They are listed in the following table. 

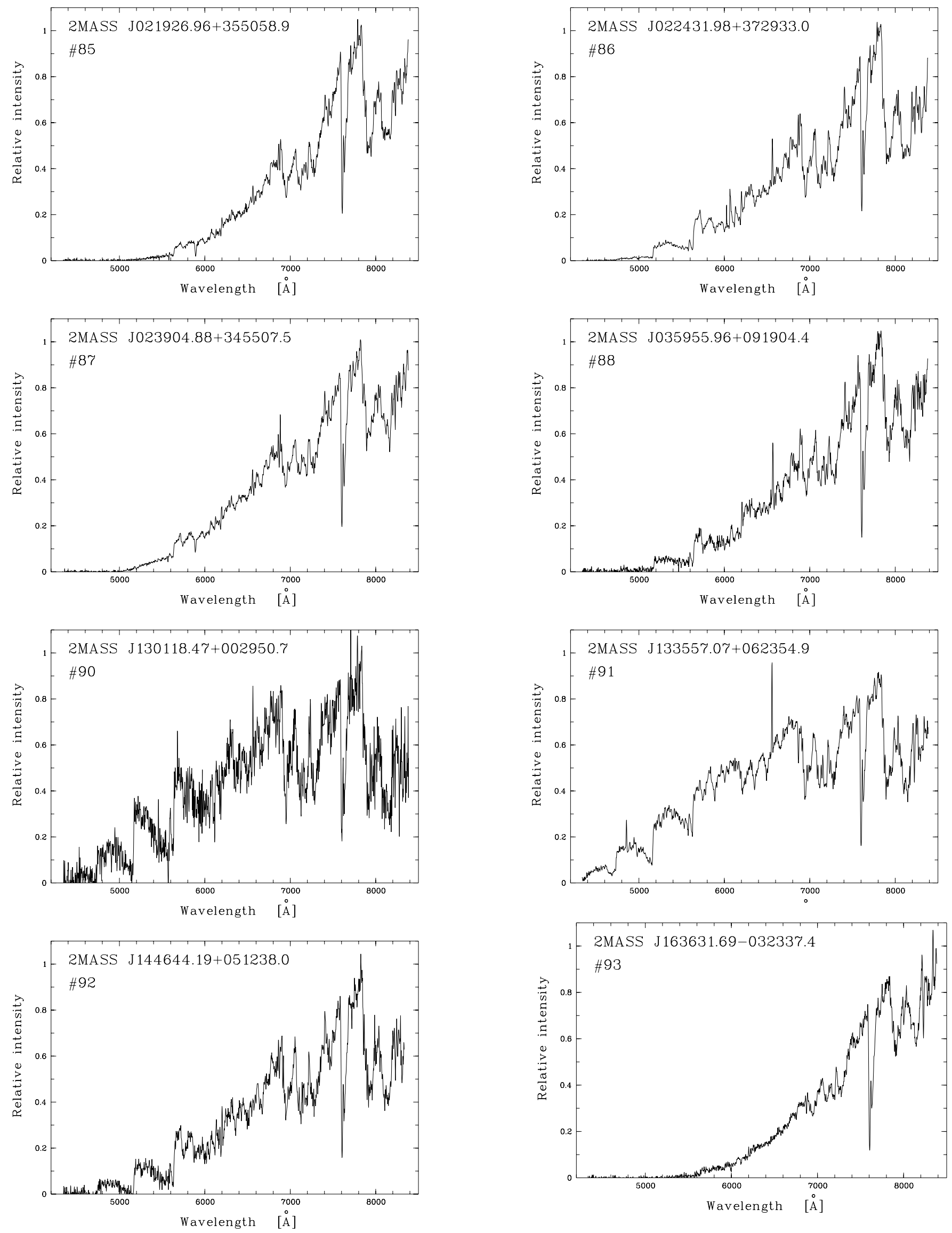

Fig. A.1. Spectra of new carbon stars in the halo. 

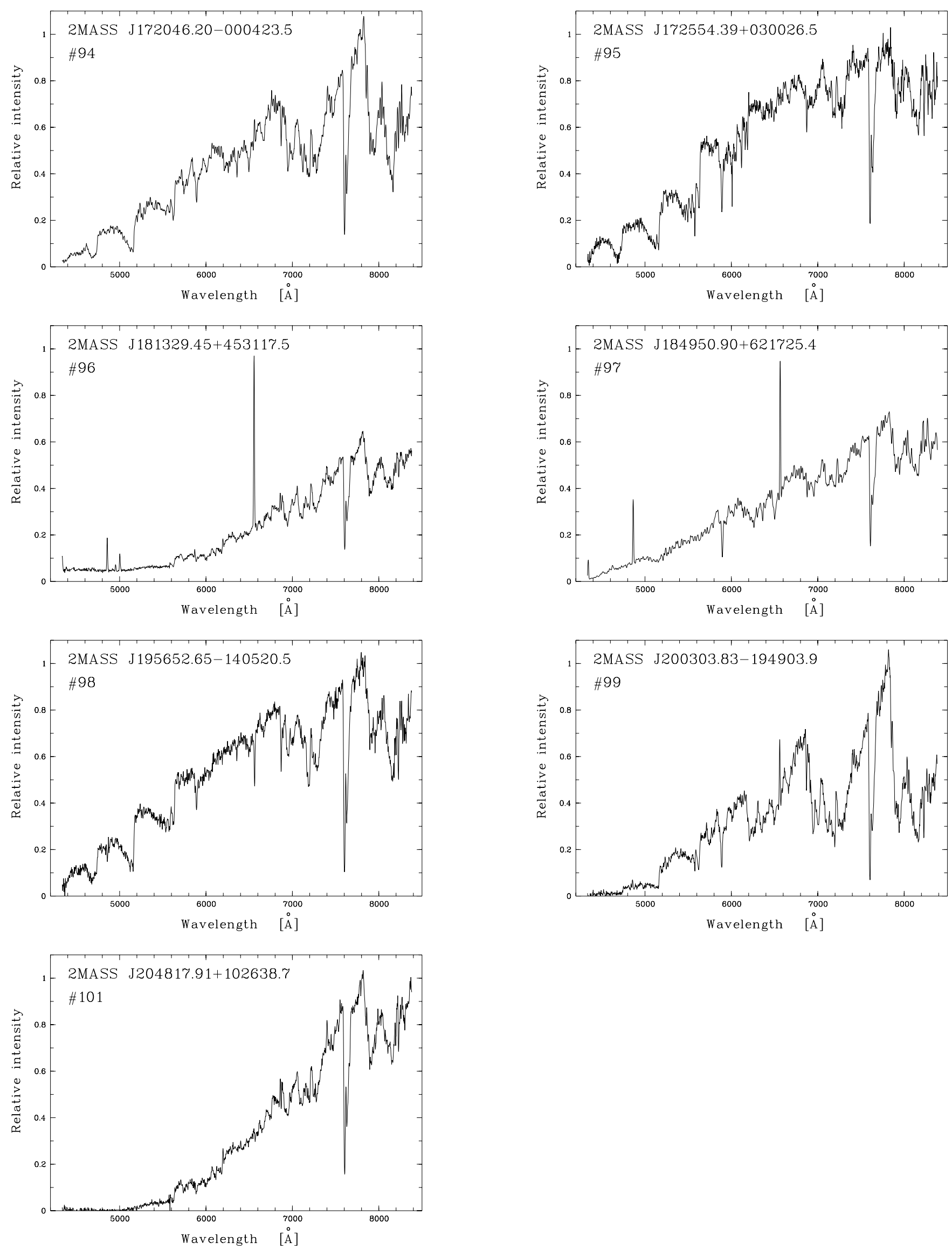

Fig. A.1. Continued. 

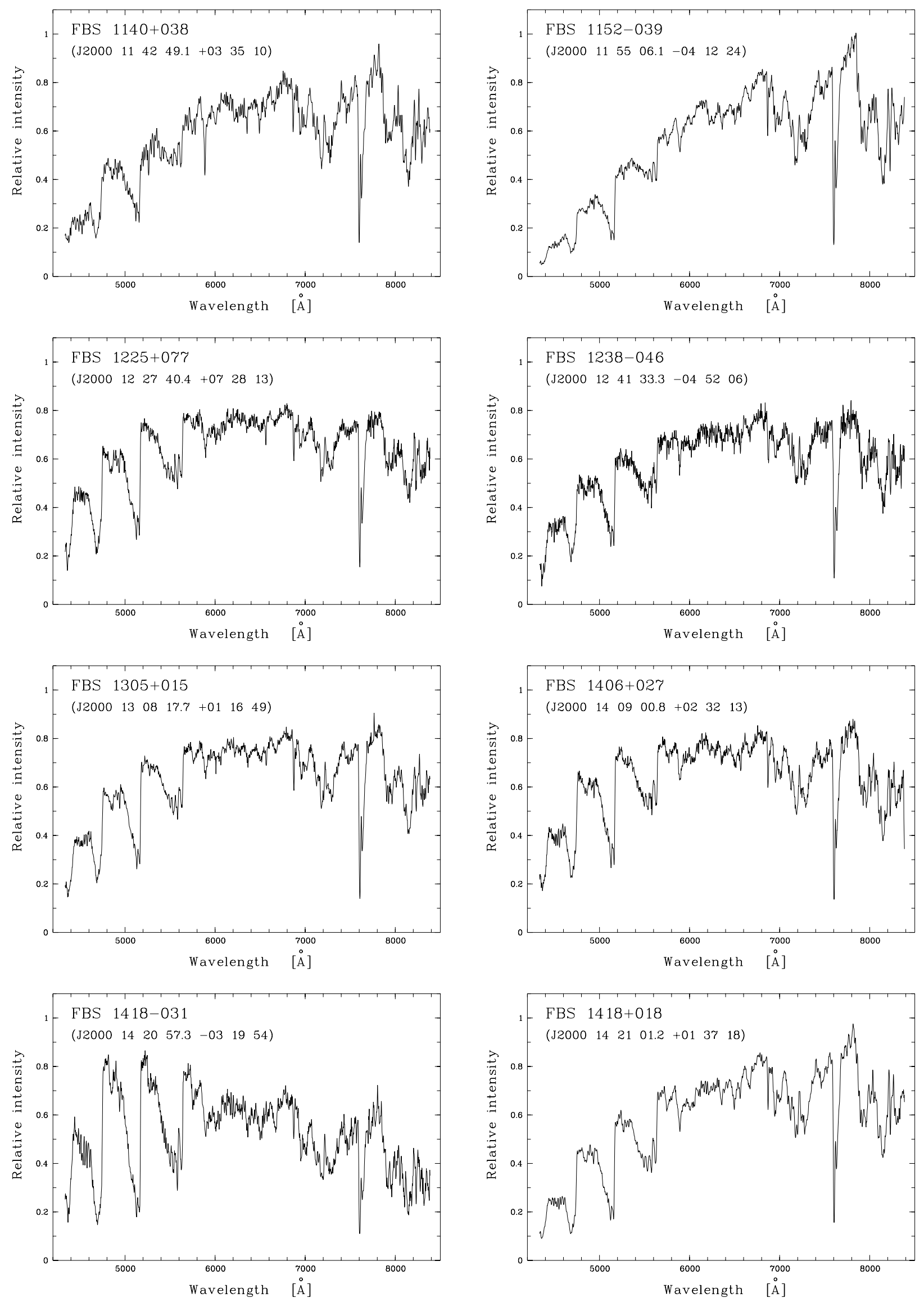

Fig. A.2. Spectra of FBS candidate carbon stars. 

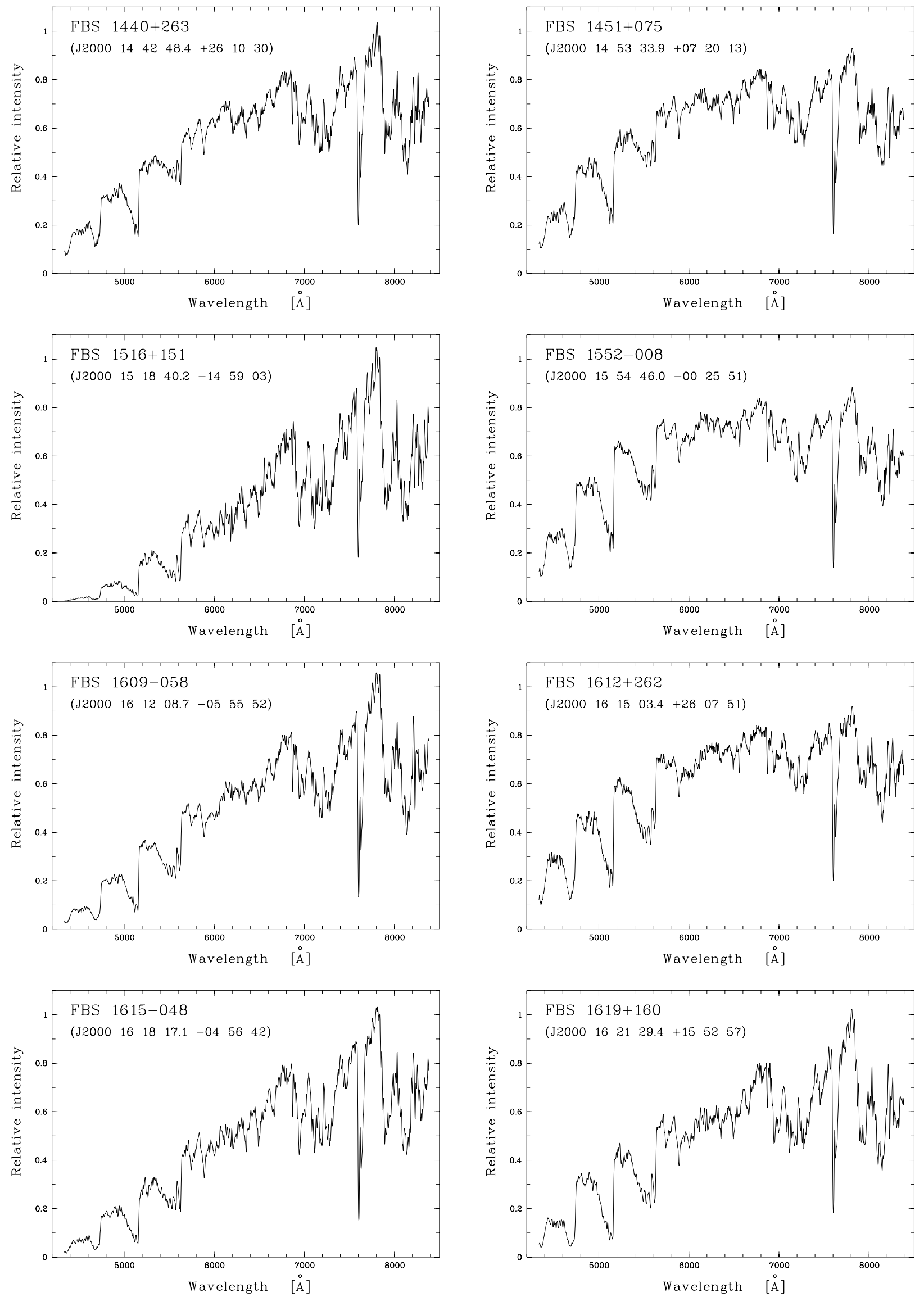

Fig. A.2. Continued. 

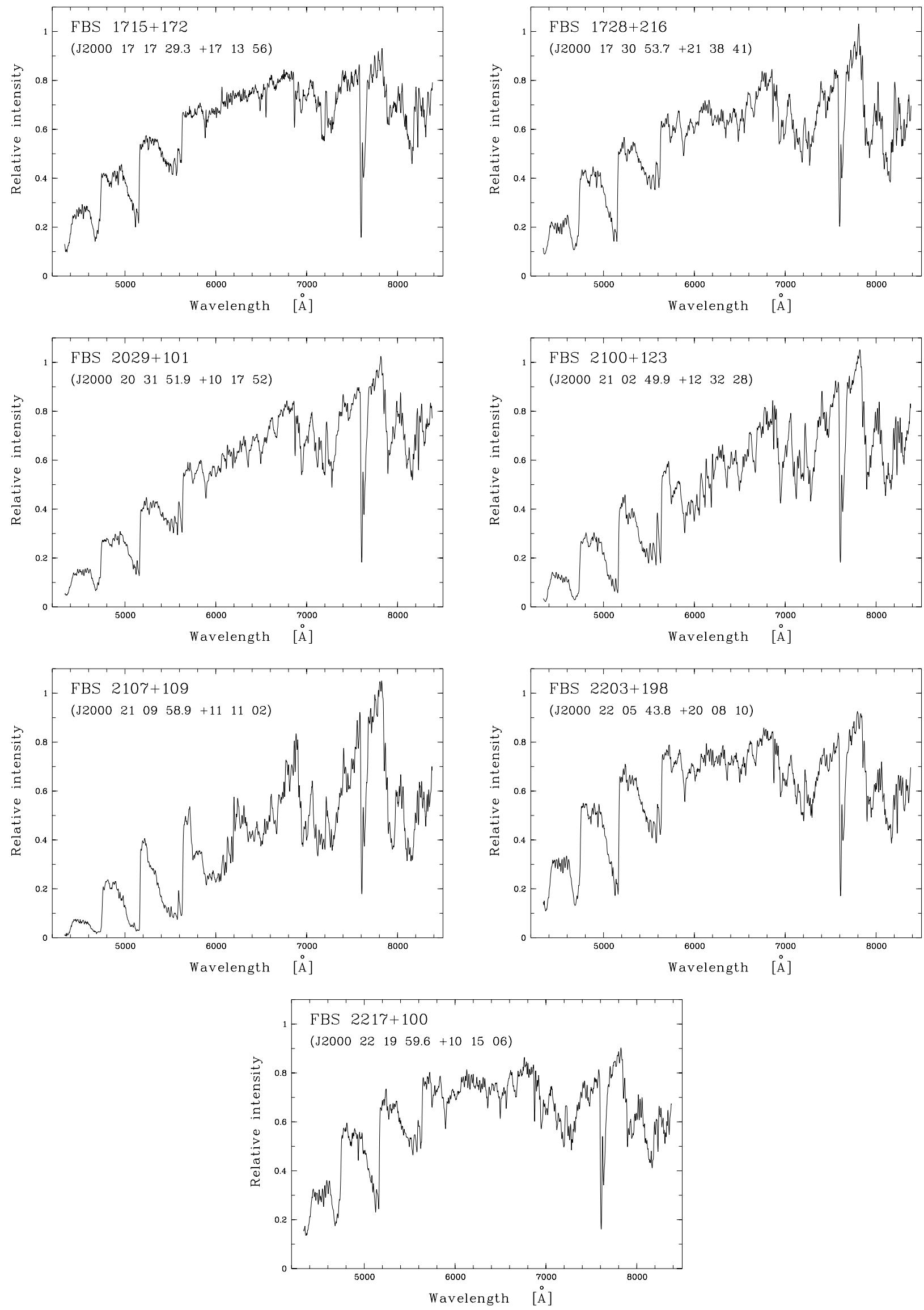

Fig. A.2. Continued. 
Table A.1. Carbon star candidates from the FBS found to be M dwarfs.

\begin{tabular}{lc}
\hline \hline Name & Coordinates J2000 \\
\hline FBS 0300-030 & $030303.2-025112$ \\
FBS 0310+016 & $031239.9+015142$ \\
FBS 0310+043 & $031319.5+043025$ \\
FBS 0318+048A & $032103.7+050329$ \\
FBS 0318+048B & $032107.2+050233$ \\
FBS 0322+041 & $032527.2+041601$ \\
FBS 1058+083 & $110116.5+080231$ \\
FBS 1435-092 & $143746.4-092659$ \\
FBS 2143-081 & $214638.0-075311$ \\
FBS 2144-089 & $214640.6-084105$ \\
\hline
\end{tabular}

\section{References}

Alksnis, A., Balklavs, A., Dzervitis, U., et al. 2001, Baltic Astron., 10, 1, CDS catalogue III/227

An, D., Terndrup, D. M., \& Pinsonneault, M. H. 2007, ApJ, 671, 1640

Beichman, C., Neugebauer, G., Habing, H. J., \& the Joint IRAS SWG 1988, The IRAS catalogue of Point Sources, version 2.0, NASA RP-1190, CDS catalogue II/125

Bergeat, J., Knapik, A., \& Rutily, B. 2002, A\&A, 385,94

Bothun G. L., Elias J. H., MacAlpine, G., et al. 1991, AJ, 101, 2220

Buchanan, C. L., Kastner, J. H., Forrest, W. J., et al. 2006, AJ, 132, 1890

Claussen, M. J., Kleinmann, S. G., Joyce, R. R., \& Jura, M. 1987, ApJS, 65,385

Clementini, G., Gratton, R., Bragaglia, A., et al. 2003, AJ, 125, 1309

Cruz, K. L., Reid, I. N., Kirkpatrick, J. D., et al. 2007, AJ,133, 439

Cutri, R. M., Skrutskie, M. F., Van Dyk, S., et al. 2003, The 2MASS All-Sky

Catalogue of Point Sources, Univ. of Massachusetts \& Infrared Processing \& Analysis Center, CDS catalogue II/246

Dahlmark, L. 1998, IBVS 4642

Downes, R. A., Margon, B., Anderson, S. F., et al. 2004, AJ, 127, 2838

Epchtein, N., Le Bertre, T., \& Lépine, J. R. D. 1990, A\&A, 227, 82

Feast, M. W., Glass, I. S., Whitelock, P. A., \& Catchpole, R. M. 1989, MNRAS, 241,375

Gigoyan, K., Mauron, N., Azzopardi, M., Muratorio, G., \& Abrahamyan, H. V. 2001, A\&A, 371, 560

Groenewegen, M. A. T., de Jong, T., van der Bliek, N. S., Slijkhuis, S., \& Willems, F. J. 1992, A\&A, 253, 150

Groenewegen, M. A. T., Oudmaijer, R. D., \& Ludwig, H. G. 1997, MNRAS, 292,686

Ibata, R., Lewis, G. F., Irwin, M., Totten, E., \& Quinn, T. 2001, ApJ, 551, 294
Jura, M. 1986, ApJ, 303, 327

Jura, M. 1987, ApJ, 313,743

Kastner, J. H., Thorndike, S. L., Romanczyk, P. A., et al. 2007, [arXiv: astro-ph/0703584v2]

Kazarovets, E. V., Samus, N. N., \& Durlevich, O. V. 2000, IBVS, 4870

Kontizas, E., Dapergolas, A., Morgan, D. H., \& Kontizas, M. 2001, A\&A, 369, 932

Law, D. R., Johnston, K. V., \& Majewski, S. R. 2005, ApJ, 619, 807

Little-Marenin, I. R., Ramsay, M. E., Stephenson, C. B., et al. 1987, AJ, 93, 663 MacConnell, D. J., 2003, PASP 115, 351

Majewski, S. R., Skrutskie, M. F., Weinberg, M. D., \& Ostheimer, J. C. 2003, ApJ, 599, 1082

Matsuura, M., Zijlstra, A. A., Bernard-Salas, J., et al. 2007, MNRAS, 382, 1889 Mauron, N., Azzopardi, M., Gigoyan, K., \& Kendall, T. R. 2004, A\&A, 418, 77

Mauron, N., Kendall, T. R., \& Gigoyan, K. 2005, A\&A, 438, 867

Mauron, N., Gigoyan, K. S., \& Kendall, T. R. 2007a, A\&A, 463, 969

Mauron, N., Gigoyan, K. S., \& Kendall, T. R. 2007b, A\&A, 475, 843

Monet, D., Bird, A., Canzian, B., et al. 1998, The USNO-A2.0 Catalogue, US Naval Observatory Flagstaff Station and Universities Space Research Association, CDS catalogue I/252

Monet, D. G., Levine, S. E., Canzian, B., et al. 2003, AJ, 125, 984

Moshir, M., Kopan, G., Conrow, T., et al. 1989, The IRAS Faint Source Catalogue, $|b|>10$, Version 2.0, CDS catalogue II/156A

Newberg, H. J., Yanny, B., Grebel, E. K., et al. 2003, ApJ, 596, L191

Nikolaev, S., \& Weinberg, M. D. 2000, ApJ, 542, 804 (NW00)

Olofsson, H. 2004, Circumstellar Envelopes, in AGB stars, ed. H. Habing, \& H. Olofsson (New-York: Springer Verlag), 330

Schlegel, D. J., Finkbeiner, D. P., \& Davis M. 1998, ApJ, 500, 525

Totten, E. J., \& Irwin M. J. 1998, MNRAS, 294, 1

Totten, E. J., Irwin, M. J., \& Whitelock, P. A. 2000, MNRAS, 314, 630

Van den Bergh, S. 2000, The Galaxies of the Local Group (Cambridge: University Press)

van Leeuwen, F., Feast, M. W., Whitelock, P. A., \& Laney, C. D. 2007, MNRAS, 379,723

Van Loon, J. T., Groenewegen, M. A. T., de Koter, A., et al. 1999, A\&A, 351, 559

Wallerstein, G., \& Knapp, G. R. 1998, ARA\&A, 36, 369

Whitelock, P. A., Menzies, J., Irwin, M. J., \& Feast, M. W. 1999, in The Stellar Content of Local Group Galaxies, Proceedings of the 192nd IAU Symp., ed. P. A. Whitelock, \& R. Cannon (ASP), 136

Whitelock, P. A., Feast, M. W., van Loon, J. T., \& Zijlstra, A. A. 2003, MNRAS, 342, 86

Whitelock, P. A., Feast, M. W., Marang, F., \& Groenewegen, M. A. T. 2006, MNRAS, 369, 751

Wood, P. R., Whiteoak, J. B., Hugues, S. M. G., et al. 1992, ApJ, 397, 552

Zijlstra, A. 2004, MNRAS, 348, L23 